\title{
Hubs-attracting Laplacian and Related Synchronization on Networks*
}

\author{
Lucia Valentina Gambuzza ${ }^{\dagger}$, Mattia Frasca ${ }^{\ddagger}$, and Ernesto Estrada ${ }^{\S}$
}

\begin{abstract}
In this work, we introduce a new Laplacian matrix, referred to as the hubs-attracting Laplacian, accounting for diffusion processes on networks where the hopping of a particle occurs with higher probability from low to high degree nodes. This notion complements the one of the hubs-repelling Laplacian discussed in [E. Estrada, Linear Algebra Appl., 596 (2020), pp. 256-280], that considers the opposite scenario, with higher hopping probabilities from high to low degree nodes. We formulate a model of oscillators coupled through the new Laplacian and study the synchronizability of the network through the analysis of the spectrum of the Laplacian. We discuss analytical results providing bounds for the quantities of interest for synchronization and computational results showing that the hubs-attracting Laplacian generally has better synchronizability properties when compared to the classical one, with a low occurrence rate for the graphs where this is not true. Finally, two illustrative case studies of synchronization through the hubs-attracting Laplacian are considered.
\end{abstract}

Key words. Laplacian matrix, networks of coupled dynamical systems, synchronization

AMS subject classifications. 05C90, 34D06, 34C15

DOI. $10.1137 / 19 \mathrm{M} 1287663$

1. Introduction. Many dynamical processes, such as flow of information in social networks, transport, epidemic spreading, and synchronization, take place on systems formed by interacting agents and are, therefore, described by mathematical models based on graphs $[1,29,19]$. The key ingredient of these models is the discrete Laplacian operator, which enables a mathematical formulation of the way in which agents interact in a network and on the evolution of the system behavior with time. The classical Laplacian (for a formal definition see section 2) is used to describe dynamical processes in which the interaction between the nodes takes place through the edges giving exactly the same weight to every edge incident with a node. An ubiquitous process in nature and society which is typically described by this graph-theoretic operator is synchronization. In synchronization processes, taking place, for instance, in neural signaling, the beating of the heart and the synchronization of fire-fly light waves, the influence of a node to each of its nearest neighbors is not depending on the topological (structural) properties of

\footnotetext{
${ }^{*}$ Received by the editors September 16, 2019; accepted for publication (in revised form) by L. DeVille February 5, 2020; published electronically May 4, 2020.

https://doi.org/10.1137/19M1287663

Funding: This work was partially supported by the University of Catania under the framework of "Piano della ricerca 201618, Linea intervento 2".

${ }^{\dagger}$ Department of Electrical, Electronic, and Computer Engineering, University of Catania, 95125 Catania, Italy (lucia.gambuzza@dieei.unict.it).

${ }^{\ddagger}$ Department of Electrical, Electronic, and Computer Engineering, University of Catania, 95125 Catania, Italy, and CNR-IASI, Italian National Research Council - Institute for Systems Analysis and Computer Science "A. Ruberti", 00185 Rome, Italy (mattia.frasca@dieei.unict.it).

$\S$ Institute of Applied Mathematics (IUMA), Universidad de Zaragoza, E-50009 Zaragoza, Spain, and ARAID Foundation, Government of Aragón, 50018 Zaragoza, Spain (estrada66@unizar.es).
}

1057 
those neighbors. That is, if the coupling strength between every pair of nodes is identical, the influence of a node to every one of its neighbors is the same. However, in the case of other diffusive dynamics, like in random walks on graphs, strategies for biasing the influence of a node on its nearest neighbors have been proposed in the literature. They are known as "degree-biased random walks" and use several strategies to bias the random walker towards high or low degree nodes $[4,23,9,3]$. In order to implement such degree-biased strategies in general dynamical systems on graphs/networks, we need the extension of the Laplacian operator to describe such scenarios.

A research agenda to generalize the Laplacian operator on graphs to describe degreebiased strategies on network dynamics was recently started by proposing the hubs-repelling Laplacian [8]. In this case a diffusive process in which the diffusive particle is biased towards low-degree nodes was described. In particular, the hubs-repelling Laplacian favors the hopping from high to low degree nodes, in such a way that navigation avoids the major hubs of the system. This hubs-repelling dynamic is inspired by processes in which such bias towards low degree nodes exists, such as diffusion in the brain or the propagation of flight delays. Here we continue the extension of the Laplacian operators to capture degree-biased mechanisms which may play a fundamental role in real-life processes. In particular, our main goal is twofold: (i) to propose a new Laplacian operator for graphs which accounts for dynamical processes biased towards high-degree nodes, and (ii) to improve the network synchronizability by using such new strategy. Synchronizability is an important, widely investigated property of the system, measuring whether a region of values of the coupling coefficient leading to synchronization (i.e., all nodes following the same trajectory) exists and how large it is. Its relation with the Laplacian spectrum and network structure is important to understand this dynamical process on networks $[14,28]$. Then, our current work is framed on the many efforts made to improve the network synchronizability proposed in recent years. Most of these methods are based on the addition/removal of links of the original structure $[26,6,27]$ or on their rewiring [32, 13], whereas others are based on changing the weights of the original structure [24], eventually in an adaptive way [16].

Here we introduce the hubs-attracting Laplacian as a graph operator defined on a Hilbert space and study some of its most important properties. In particular, as the synchronization properties depend on the spectrum of the Laplacian, we provide analytical and computational results characterizing the spectrum of this new operator. We prove that this spectrum is real and nonnegative, although the hubs-attracting Laplacian is nonsymmetric. As a major result, we find that the hubs-attracting Laplacian generally improves the synchronizability of a network. The hubs-attracting Laplacian here introduced can be viewed as a method to change the network weights to improve its synchronizability. Our results show that, although there are cases where synchronizability is not enhanced, they are characterized by a low probability of occurrence.

The rest of the paper is organized as follows: preliminaries are given in section 2; the hubs-attracting Laplacian is introduced in section 3; the model of oscillators coupled through this Laplacian is presented in section 4, and the spectrum of the "hubs-attracting Laplacian" is studied in section 5. In section 6 two case studies of systems of units interacting through the "hubs-attracting Laplacian" are discussed. Finally, in section 7 the conclusions of the paper are drawn. 
2. Preliminaries. Here we use interchangeably the terms graphs and networks. A simple, undirected graph $G=(V, E)$ is formed by the set of vertices or nodes $V$ and the set of edges $E$. We will always consider connected graphs. If $G$ is disconnected, we consider a componentwise analysis of the graph. In the case of weighted graphs $G=(V, E, W, \varphi)$, there is a surjective mapping $\varphi: E \rightarrow W$ assigning elements of the weight set $W$ to the edges of the graph. Here we consider only positive weights. Let $A$ be the adjacency matrix of the (weighted) graph $G$, and let $k_{i}$ denote the degree of the node $i \in V$, i.e., the sum of the $i$ th row or column of $A$. In the case of weighted graphs the degree is usually referred to as strength, but we will use the general term degree here in all cases. Let $i \in V$ be a node of $G$. We will denote by $\mathcal{N}_{i}=\{j \in V \mid(i, j) \in E\}$ the set of all nearest neighbors of $i$. A node for which $k_{i} \gg 1$ will be "informally" called a hub. We will denote by $K$ the diagonal matrix of node degrees.

Let $\ell^{2}(V)$ be the Hilbert space of square-summable functions on $V$ with inner product

$$
\langle f, g\rangle=\sum_{v \in V} f(v) \overline{g(v)}, \quad f, g \in \ell^{2}(V) .
$$

The graph Laplacian $[20,22,25,12,11,31]$ is an operator in $\ell^{2}(V)$ which is defined by

$$
(L f)(v):=\sum_{w \in V:(v, w) \in E} \rho_{v w}(f(v)-f(w)), \quad f \in \ell^{2}(V),
$$

where $\rho_{v w} \in W$. The Laplacian matrix can be obtained as $L=K-A$. We remind the reader that in the case of weighted graphs $K$ is the diagonal matrix of weighted degrees and $A$ is the weighted adjacency matrix.

\section{Hubs-attracting Laplacian.}

Definition 1. The hubs-attracting Laplacian for a connected graph is an operator in $\ell^{2}(V)$ which is defined by

$$
\left(L_{A} f\right)(v):=\sum_{w \in \mathcal{N}_{v}} \frac{k_{w}}{k_{v}}(f(v)-f(w)), \quad f \in \ell^{2}(V) .
$$

Let us designate $\varpi_{v, w}=\frac{k_{w}}{k_{v}}$ as the edge weight for the edge $(v, w) \in E$. Let $e_{v}, v \in V$ be a standard orthonormal basis in $\ell^{2}(V)$ consisting of the vectors

$$
e_{v}(w):= \begin{cases}1 & \text { if } w=v \\ 0 & \text { otherwise. }\end{cases}
$$

Then, $L_{A}$ acts on the vectors $e_{v}$ as follows:

$$
\left(L_{A} e_{v}\right)(w)= \begin{cases}\kappa_{A}(v) & \text { if } w=v \\ -\varpi_{v, w} & \text { if }(v, w) \in E \\ 0 & \text { otherwise }\end{cases}
$$

where 
(5)

$$
\kappa_{A}(v)=\sum_{w \in \mathcal{N}_{v}} \varpi_{v, w}
$$

Let $\Xi$ be a diagonal matrix with $\Xi_{v, v}=\kappa_{A}(v)$ and $\Xi_{v, w}=0$ for all $v \neq w$. Then,

$$
L_{A}=\Xi-K^{-1} A K
$$

The counterpart of the hubs-attracting Laplacian is the hubs-repelling Laplacian, introduced in [8] and defined by the following definition.

Definition 2. The hubs-repelling Laplacian for a connected graph is an operator in $\ell^{2}(V)$ which is defined by

$$
\left(L_{R} f\right)(v):=\sum_{w \in \mathcal{N}_{v}} \frac{k_{v}}{k_{w}}(f(v)-f(w)), \quad f \in \ell^{2}(V) .
$$

Some examples of $L_{A}$ along with the corresponding $L$ and $L_{R}$ are shown in Table 1 . We will compare the use of the different Laplacians for the specific examples of synchronization dealt with in section 6 .

Table 1

Illustration of the hubs-attracting $L_{A}$, repelling $L_{R}$, and classic Laplacian $L$ in two simple undirected graphs.

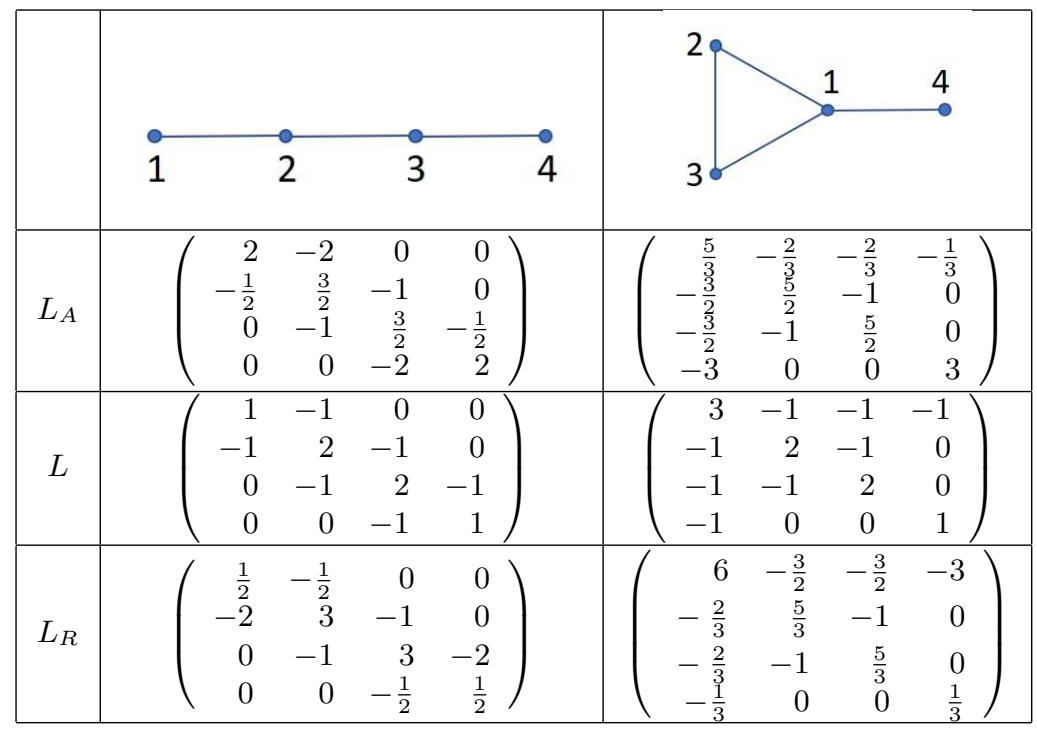

It is clear that the hubs-attracting Laplacian induces a representation of the graph as a weighted directed graph $\mathcal{D}(G)=(V, \hat{E})$, such that if $(u, v) \in E$, then $(u \rightarrow v) \in \hat{E}$ with weight $k_{v} / k_{u}$ and $(v \rightarrow u) \in \hat{E}$ with weight $k_{u} / k_{v}$. The most important consequence of this is that $L_{A}$ has some nice properties as proved in the following results.

Theorem 3. The hubs-attracting Laplacian matrix $L_{A}$ has the following properties: 
(i) its eigenvalues are real;

(ii) it is positive semidefinite;

(iii) rank $L_{A}=n-c$, where $c$ is the number of connected components;

(iv) it can be diagonalized as $L_{A}=(K U) \Lambda(K U)^{-1}$, where $\Xi-A=U \Lambda U^{-1}$ and $K$ is the diagonal matrix of node degrees.

Proof. We start by showing that

$$
L_{A}=\Xi-K^{-1} A K=K^{-1}\left(K \Xi K^{-1}-A\right) K=K^{-1}(\Xi-A) K,
$$

thus, $L_{A}$ is similar to a symmetric matrix, so its eigenvalues are real.

Now let $\vec{x} \in \mathbb{R}^{n}, \vec{x} \neq \overrightarrow{0}$. Then, we can write

$$
\vec{x}^{T}(\Xi-A) \vec{x}=\sum_{(i, j) \in E}\left(x_{i} \sqrt{\frac{k_{j}}{k_{i}}}-x_{j} \sqrt{\frac{k_{i}}{k_{j}}}\right)^{2} .
$$

Then, because $\Xi-A$ and $L_{A}$ are similar, we also have that $\vec{x}^{T} L_{A} \vec{x} \geq 0$.

Let us now prove that the dimension of the null space of $L_{A}$ is $c$. Let $\vec{z}$ be a vector such that $L_{A} \vec{z}=0$. This implies that for every $(i, j) \in E, z_{i}=z_{j}$. Therefore $\vec{z}$ takes the same value on all nodes of the same connected component, which indicates that the dimension of the null space is $c$, and so rank $L_{A}=n-c$. Finally, because $\Xi-A$ is symmetric we can write it as $\Xi-A=U \Lambda U^{-1}$. Thus, $L_{A}=(K U) \Lambda(K U)^{-1}$ indicating that the hubs-repelling Laplacian is diagonalizable.

It is also important to note that in a regular graph the ratios $k_{v} / k_{u}$ and $k_{u} / k_{v}$ are equal to one for every pair of nodes, which means that the underlying graph $\mathcal{D}(G)$ of any undirected graph is just an unweighted graph. Thus, we have the following result.

Lemma 4. Let $G$ be a k-regular graph. Then

$$
L_{A}=L=k I-A .
$$

4. Synchronization in networks of oscillators coupled through the hubs-attracting Laplacian. Dynamical oscillators interacting through diffusive coupling are modeled by the following set of ordinary differential equations:

$$
\dot{x}_{i}=f\left(x_{i}\right)-\sigma \sum_{j=1}^{N} L_{i j} H x_{j},
$$

with $i=1, \ldots, N$ and where $x_{i} \in \mathbb{R}^{n}$ is the vector of state variables of the oscillator at node $i$, $f\left(x_{i}\right)$ the local (uncoupled) dynamics, $\sigma$ the coupling coefficient, $H$ the inner coupling matrix with $\{0,1\}$ elements selecting the variables involved in coupling, and $L$ the classical Laplacian.

Here, we propose a generalization of (10) replacing the classical Laplacian with the hubsattracting Laplacian:

$$
\dot{x}_{i}=f\left(x_{i}\right)-\sigma \sum_{j=1}^{N} L_{A, i j} H x_{j},
$$

Copyright $\odot$ by SIAM. Unauthorized reproduction of this article is prohibited. 


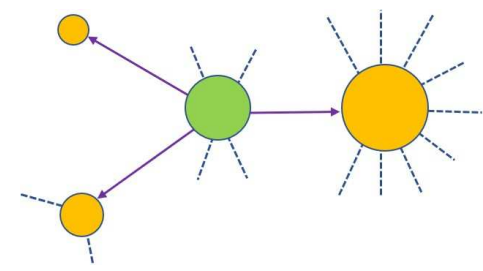

(a)

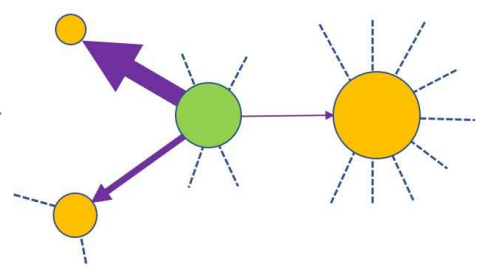

(b)

Figure 1. Schematic illustration of the "interaction" processes controlled by the normal Laplacian (a) and the hubs-attracting Laplacian (b).

where $i=1, \ldots, N$. Equation (11) can be rewritten as

$$
\dot{x}_{i}=f\left(x_{i}\right)+\sigma \sum_{j=1}^{N} \frac{k_{j}}{k_{i}} a_{i j} H\left(x_{j}-x_{i}\right),
$$

where $a_{i j}$ are the coefficients of the adjacency matrix $A$.

From this expression, we note that high-degree nodes receive weaker influence from their neighbors, compared to low-degree nodes. In addition, an influence from a neighbor with high degree has a stronger weight than that coming from a neighbor with lower degree. These asymmetric influences are schematically illustrated in Figure 1.

We say that a network of coupled oscillators synchronizes when $\lim _{t \rightarrow \infty}\left\|x_{i}-x_{j}\right\|=0$ for $i, j=1, \ldots, N$. The onset of synchronization depends on many factors, such as the dynamics of the units, the topology of the network, and the coupling coefficient [2, 1]. Synchronizability is a property measuring the existence of a region of values of the coupling coefficient leading to synchronization and its extent. The synchronizability of system (10) is determined by the eigenvalues of the Laplacian $L$. There are two types of networks with bounded and unbounded synchronization regions in the parameter space. One large class of dynamic networks has an unbounded synchronized region specified by

$$
\sigma \mu_{2}>\alpha_{1}>0
$$

where the constant $\alpha_{1}$ depends only on the node dynamics. Here, a bigger spectral gap $\mu_{2}$ implies a better network synchronizability, namely a smaller coupling strength $\sigma>0$ is needed $[5,6,7,18]$.

Another large class of dynamic networks has a bounded synchronized region specified by

$$
\sigma \mu_{2}, \ldots, \sigma \mu_{N} \in\left(\alpha_{2}, \alpha_{3}\right) \subset(0, \infty),
$$

where the constants $\alpha_{2}, \alpha_{3}$ depend only on the node dynamics as well, and a bigger eigenratio $Q \triangleq \mu_{2} / \mu_{N}$ implies a better network synchronizability, which likewise means a smaller coupling strength is needed $[30,15]$.

These criteria straightforwardly generalize to systems of oscillators coupled through other Laplacians as in (11). Taking into account that $L_{A}$ has real eigenvalues, it is possible to define 
the algebraic connectivity $\mu_{A, 2}$ and the eigenratio $Q_{A} \triangleq \mu_{A, 2} / \mu_{A, N}$ for the hubs-attracting Laplacian. Synchronization in networks of oscillators coupled through the hubs-attracting Laplacian thus crucially depends on these two quantities and, for this reason, the next section is devoted to provide some analytical and computational results on them.

\section{Spectrum of the hubs-attracting Laplacian.}

5.1. Analytical results. Let us focus our attention to the eigenvalues of $L_{A}$. Let $0=$ $\mu_{A, 1}<\mu_{A, 2} \leq \cdots \leq \mu_{A, N}$ be the eigenvalues of $L_{A}$ in a connected simple graph. Let $\tau_{A}(i)=\Xi_{i i}$, and let $\delta, \Delta$, and $\bar{k}$ be the minimum, maximum, and mean degree of a node in $G$, respectively. Then we have the following results.

Lemma 5. Let $G$ be a simple connected graph. Then,

$$
\mu_{A, N} \leq 2 \max _{i} \tau_{A}(i) \leq 2 \Delta .
$$

Proof. Using the Gershgorin circle theorem we have that $\mu_{A, N}$ is contained in a circle of center in $\tau_{A}(N)$ and $\operatorname{radius}_{\max _{i}} \tau_{A}(i)$. Thus, $\mu_{A, N} \leq 2 \max _{i} \tau_{A}(i)$. Then, $\max _{i} \tau_{A}(i)=$ $\max _{i} k_{i}^{-1} \sum_{v \in \eta_{i}} k_{v}$, the last maximum is obtained when $k_{i}=\delta$, where $\delta$ is the minimum degree, and it is connected to $\delta$ nodes $v \in \eta_{i}$ which all have the maximum degree $\Delta$. Thus, the result follows.

Lemma 6. Let $G$ be a simple connected graph with $N \geq 2$ nodes and $M$ links. Then,

$$
\mu_{A, N} \geq \frac{1}{N-1} \sum_{(u, v) \in E} \frac{k_{u}^{2}+k_{v}^{2}}{k_{u} k_{v}} \geq \frac{2 M}{N-1}>\bar{k} .
$$

Proof. We have that $\operatorname{tr}\left(L_{A}\right)=\sum_{j=2}^{N} \mu_{A, j}$, where $\operatorname{tr}\left(L_{A}\right)$ is the trace of $L_{A}$. Thus $\mu_{A, N} \geq$ $\operatorname{tr}\left(L_{A}\right) /(N-1)$. Then, we have that

$$
\operatorname{tr}\left(L_{A}\right)=\sum_{(u, v) \in E} \frac{k_{u}^{2}+k_{v}^{2}}{k_{u} k_{v}} .
$$

Because the minimum value that $k_{i}$ can take for any node is one, $\operatorname{tr}\left(L_{A}\right)$ is upper bounded as follows:

$$
\sum_{(u, v) \in E} \frac{k_{u}^{2}+k_{v}^{2}}{k_{u} k_{v}} \geq 2 M
$$

with equality if and only if the graph is regular. Because $\bar{k}=2 M / N$, the final result then follows.

We now prove a result for the second smallest eigenvalue of the hubs-attracting Laplacian.

Lemma 7. Let $G$ be a connected graph. Then

$$
\mu_{A, 2}=\inf _{\vec{x}^{T} K \overrightarrow{1}=0} \frac{\vec{x}^{T}(\Xi-A) \vec{x}}{\vec{x}^{T} \vec{x}}=\inf _{\vec{z}^{T} \overrightarrow{1}=0} \frac{\vec{z}^{T}\left(K^{-1} \Xi K^{-1}-K^{-1} A K^{-1}\right) \vec{z}}{\vec{z}^{T} K^{-2} \vec{z}},
$$

where $\vec{x}, \vec{z} \in \mathbb{R}^{n}, \vec{x}, \vec{z} \neq \overrightarrow{0}$. 
Theorem 8. Let $u \in V$ and $v \in V$ be two nodes of the graph $G=(V, E)$ such that $(u, v) \notin$ E. Then,

$$
\mu_{A, 2} \leq \frac{k_{u}^{-3} \sum_{i \in \mathcal{N}_{u}} k_{i}+k_{v}^{-3} \sum_{j \in \mathcal{N}_{v}} k_{j}}{k_{u}^{-2}+k_{v}^{-2}}=\frac{\frac{k_{v}^{2}}{k_{u}} \sum_{i \in \mathcal{N}_{u}} k_{i}+\frac{k_{u}^{2}}{k_{v}} \sum_{j \in \mathcal{N}_{v}} k_{j}}{k_{u}^{2}+k_{v}^{2}}
$$

Proof. Let

$$
\vec{z}=\vec{e}_{u}-\vec{e}_{v}=[0, \ldots 0, \underbrace{1}_{u}, 0, \ldots, 0, \underbrace{-1}_{v}, 0, \ldots 0]^{T} .
$$

It is easy to see that $\vec{z}^{T} K^{-2} \vec{z}=k_{u}^{-2}+k_{v}^{-2}$ and $\vec{z}^{T} \overrightarrow{1}=0$. It can be seen that $\vec{z}^{T} K^{-1} \Xi K^{-1} \vec{z}=$ $k_{u}^{-2} \kappa_{A}(u)+k_{v}^{-2} \kappa_{A}(v)$ and $\vec{z}^{T} K A K \vec{z}=0$ because $(u, v) \notin E$. Therefore, using the result of Lemma 7 we have

$$
\begin{aligned}
\mu_{A, 2} & \leq \frac{\vec{z}^{T}\left(K^{-1} \Xi K^{-1}-K^{-1} A K^{-1}\right) \vec{z}}{\vec{z}^{T} K^{2} \vec{z}} \\
& =\frac{k_{u}^{-2} \kappa_{A}(u)+k_{v}^{-2} \kappa_{A}(v)}{k_{u}^{-2}+k_{v}^{-2}} \\
& =\frac{k_{u}^{-3} \sum_{i \in \mathcal{N}_{u}} k_{i}+k_{v}^{-3} \sum_{j \in \mathcal{N}_{v}} k_{j}}{k_{u}^{-2}+k_{v}^{-2}} .
\end{aligned}
$$

This completes the proof.

As discussed in section 4 , another spectral parameter of great importance for understanding the synchronization dynamics on networks is $Q_{A}=\frac{\mu_{A, 2}}{\mu_{A, N}}$. Based on the previous result we have the following nontrivial bound for the eigenratio $Q_{A}$.

Lemma 9. Let $G=(V, E)$ be a connected graph, let $u \in V, v \in V,(u, v) \notin E$, and let $M=|E|, N=|V|$, and $\bar{k}=2 M / N$. Then,

$$
\begin{aligned}
Q_{A} \leq & \frac{(N-1)\left(\frac{k_{v}^{2}}{k_{u}} \sum_{i \in \mathcal{N}_{u}} k_{i}+\frac{k_{u}^{2}}{k_{v}} \sum_{j \in \mathcal{N}_{v}} k_{j}\right)}{\left(k_{u}^{2}+k_{v}^{2}\right) \sum_{(r, s) \in E} \frac{k_{r}^{2}+k_{s}^{2}}{k_{r} k_{s}}} \\
\leq & \frac{(N-1)\left(\frac{k_{v}^{2}}{k_{u}} \sum_{i \in \mathcal{N}_{u}} k_{i}+\frac{k_{u}^{2}}{k_{v}} \sum_{j \in \mathcal{N}_{v}} k_{j}\right)}{2\left(k_{u}^{2}+k_{v}^{2}\right) M} \\
\leq & \frac{\frac{k_{v}^{2}}{k_{u}} \sum_{i \in \mathcal{N}_{u}} k_{i}+\frac{k_{u}^{2}}{k_{v}} \sum_{j \in \mathcal{N}_{v}} k_{j}}{\left(k_{u}^{2}+k_{v}^{2}\right) \bar{k}}
\end{aligned}
$$

Copyright (C) by SIAM. Unauthorized reproduction of this article is prohibited. 
Table 2

Occurrence of networks with $\mu_{A, 2}<\mu_{2}$ or with $Q_{A}<Q$ for all connected graphs with $6 \leq N \leq 10$.

\begin{tabular}{|c|c|c|c|}
\hline$n$ & Number of networks & Occurrence of $\mu_{A, 2}<\mu_{2}(\%)$ & Occurrence of $Q_{A}<Q(\%)$ \\
\hline \hline 6 & 112 & $0.89 \%$ & $0.89 \%$ \\
\hline 7 & 853 & $0.59 \%$ & $0.82 \%$ \\
\hline 8 & 11,117 & $0.32 \%$ & $0.39 \%$ \\
\hline 9 & 261,080 & $0.18 \%$ & $0.15 \%$ \\
\hline 10 & $11,716,571$ & $0.09 \%$ & $0.07 \%$ \\
\hline
\end{tabular}

\subsection{Computational results.}

5.2.1. Small graphs. We first consider all connected graphs with $2<N \leq 10$ nodes, which total 11,989,764 graphs. Although these graphs are relatively small, the big size of this data set somehow guarantees a wide structural variability as to understand some of the main factors influencing the spectral parameters of $L_{A}$, namely $\mu_{A, 2}$ and $Q_{A}$. In Figure 2 we show the results obtained for $\mu_{A, 2}$. We observe that, up to $N=5, \mu_{A, 2}$ is always greater than or equal to $\mu_{2}$. For graphs with a higher number of nodes, there are occurrences where the condition $\mu_{A, 2} \geq \mu_{2}$ is violated. For $N=6$ we have a single case, for $N=7$ five cases, and so on as reported in Table 2, showing that the percentage of such "odd" cases is quite small. Figure 2 also illustrates the bound for $\mu_{A, 2}$ provided by Theorem 8 and a comparison with the first nonzero eigenvalue of the hubs-repelling Laplacian $L_{R}$, referred to as $\mu_{R, 2}$. Here, we note that the different diffusion mechanisms at the basis of the definition of the hubs-repelling and the hubs-attracting Laplacian produce matrices with a different $\mu_{2}$, where, in particular, for almost all networks considered, we have that $\mu_{R, 2} \geq \mu_{A, 2}$ (we refer the reader to [8] for more details on the spectral properties of the hubs-repelling Laplacian).

Similarly, for the same set of networks, we compute the ratio $Q_{A}$ (Figure 3 ). We note that for $N \leq 5, Q_{A}$ is always greater than or equal to $Q$, while, for higher $N$, there are occurrences where $Q_{A} \geq Q$ is violated. Table 2 also illustrates the number of occurrences where $Q_{A}<Q$, showing again that they occur at small percentages. The comparison between $Q_{A}$ and the corresponding parameter in the hubs-repelling Laplacian, referred to as $Q_{R}$, also shows the occurrence of a general trend, with few cases where it is violated. In particular, we observe that for almost all networks considered $Q_{A} \geq Q_{R}$ and again refer the reader to [8] for a more detailed discussion on the spectral properties of the hubs-repelling Laplacian.

The analysis of the graphs showing an "odd" behavior, either in terms of $\mu_{A, 2}$ or $Q_{A}$, seems quite interesting, but at the same time challenging. The seven examples of networks with $N=7$ nodes and showing $Q_{A}<Q$ are illustrated in Figure 4; the first five of these graphs (Figure 4(a)-(e)) also show $\mu_{A, 2}<\mu_{2}$. For larger networks we have found examples where $\mu_{A, 2}<\mu_{2}$ and $Q_{A}>Q$, thus demonstrating that the "odd" behavior can refer to either one of the two quantities $\mu_{A, 2}$ or $Q_{A}$ or to both. Understanding the properties of the graph generating these behavior is left for a future work, while here we only observe that all of the cases found in these graphs have a minimum degree greater than or equal to two.

5.2.2. Large random graphs. Here we consider larger networks, focusing in particular on two paradigmatic models of random graphs: the Erdös-Rényi (ER) model and the BarabasiAlbert (BA) model. For each of them, we have generated 5000 sample networks with $N=200$

Copyright (C) by SIAM. Unauthorized reproduction of this article is prohibited. 

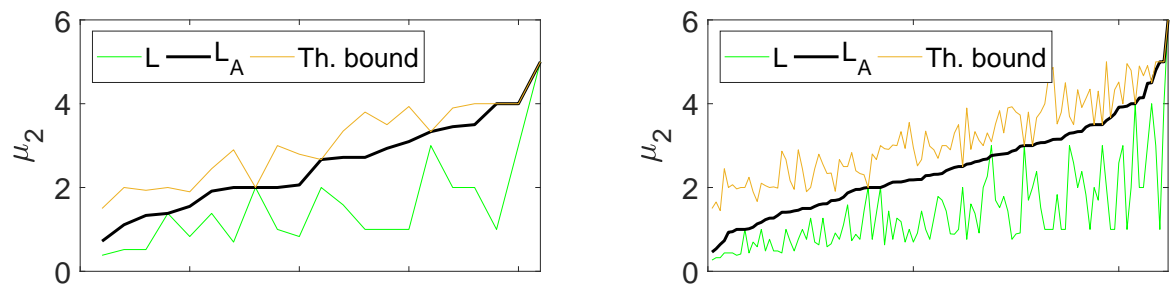

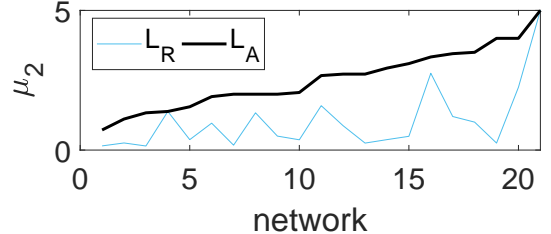

(a)
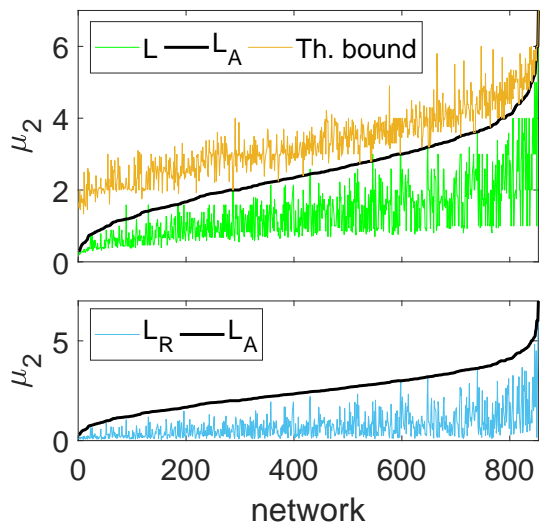

(c)

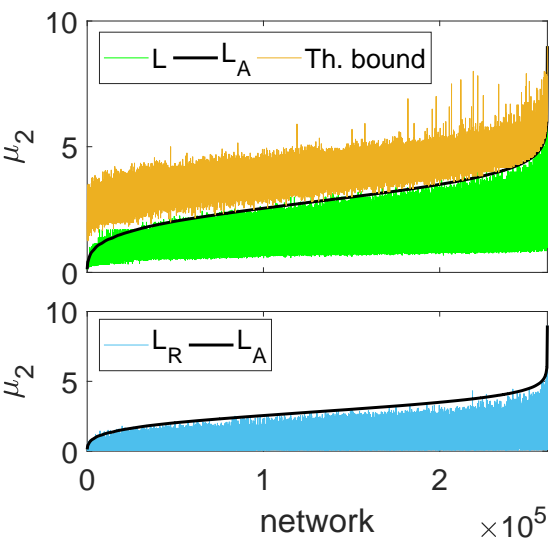

(e)

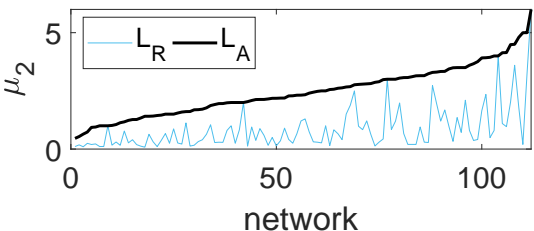

(b)
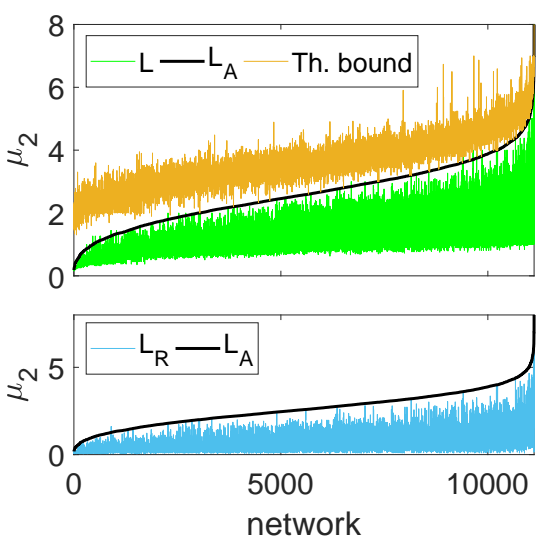

(d)

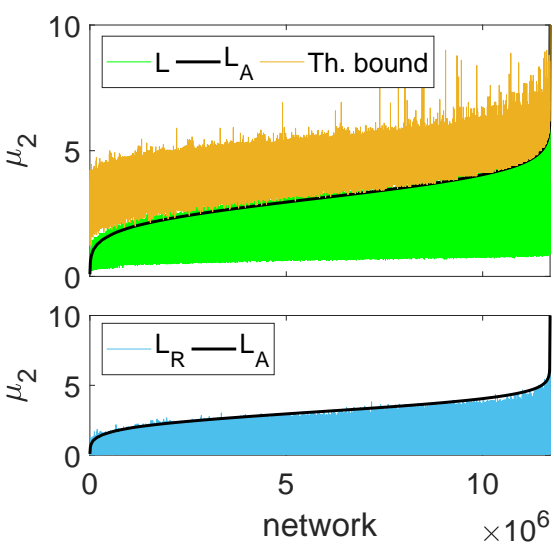

(f)

Figure 2. First nonzero eigenvalue for the classical Laplacian, $\mu_{2}$, for the hubs-attracting Laplacian, $\mu_{A, 2}$, for the hubs-repelling Laplacian, $\mu_{R, 2}$, and theoretical upper bound as in (20) for all connected graphs with given $N$ : (a) $N=5$; (b) $N=6$; (c) $N=7$; (d) $N=8$; (e) $N=9$; (f) $N=10$. For better visualization networks are reordered according to increasing values of $\mu_{A, 2}$. For each panel, the upper part illustrates $\mu_{A, 2}$, $\mu_{2}$, and the theoretical bound, while the lower part compares $\mu_{A, 2}$ with $\mu_{R, 2}$.

Copyright (c) by SIAM. Unauthorized reproduction of this article is prohibited. 

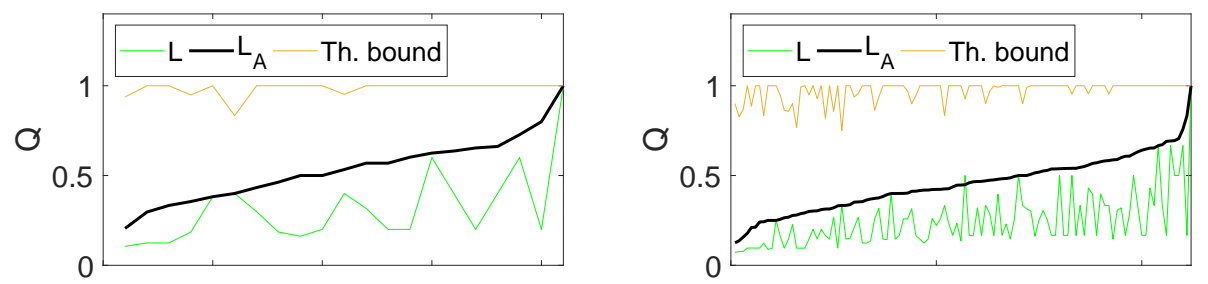

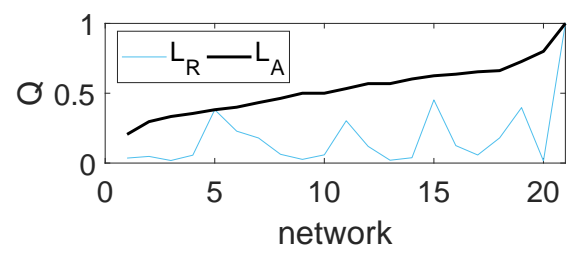

(a)
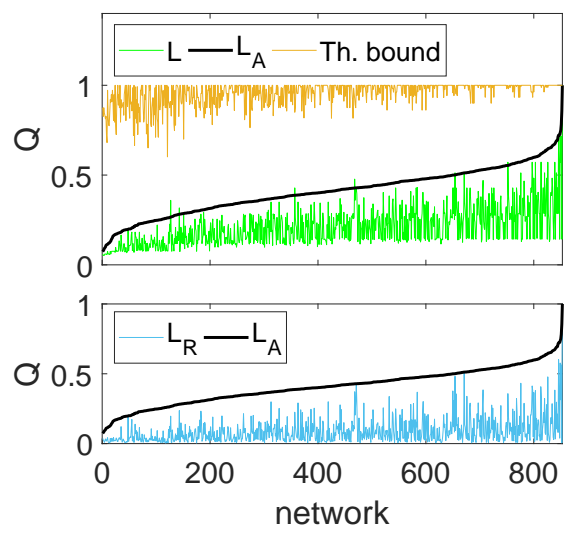

(c)

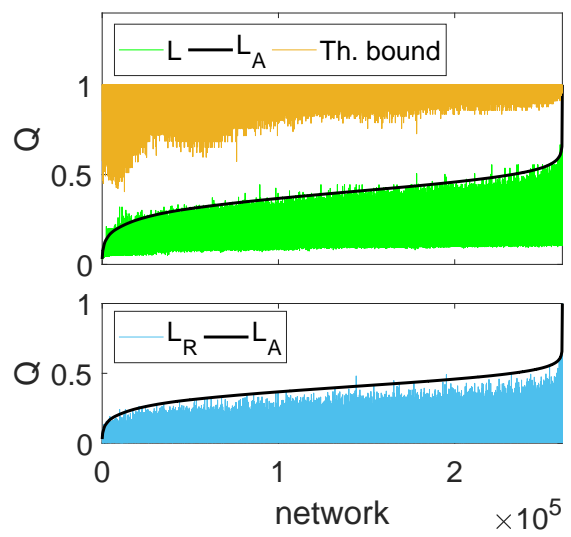

(e)

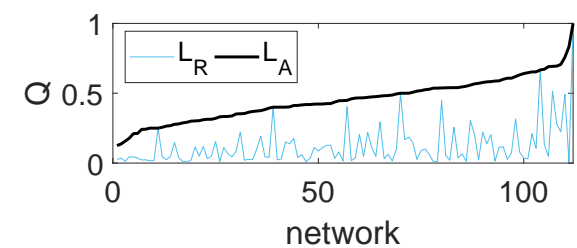

(b)

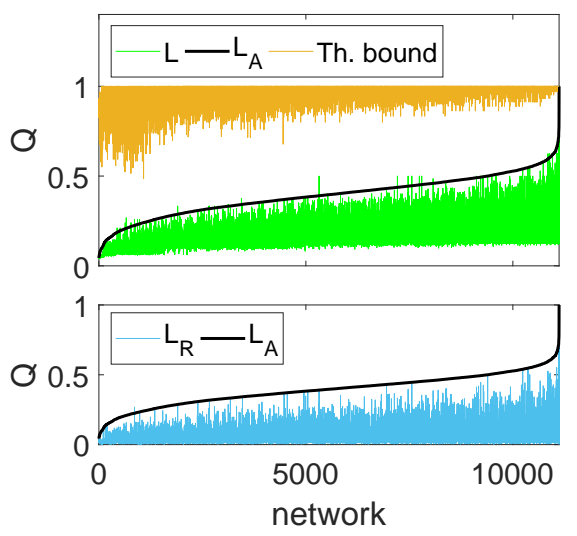

(d)
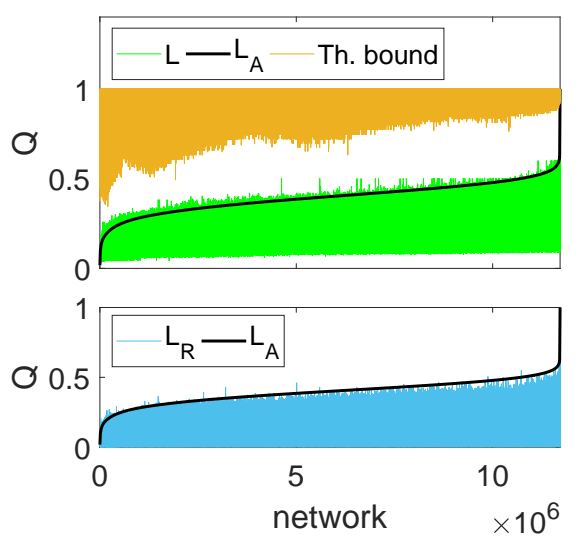

(f)

Figure 3. Ratio between the largest and smallest nonzero eigenvalue, $Q$, for the classical Laplacian, and $Q_{A}$ for the hubs-attracting Laplacian for all connected graphs with given $n:$ (a) $n=5$; (b) $n=6$; (c) $n=7$; (d) $n=8$; (e) $n=9$; (f) $n=10$. The theoretical bound is provided by (22); when this bound is trivial, i.e., $Q_{A}>1$, we simply give the nontrivial upper bound of $Q_{A}=1$ in the plot. For better visualization networks are reordered according to increasing values of $Q_{A}$. For each panel, the upper part illustrates $Q_{A}, Q$ and the theoretical bound, while the lower part compares $Q_{A}$ with $Q_{R}$.

Copyright (C) by SIAM. Unauthorized reproduction of this article is prohibited. 


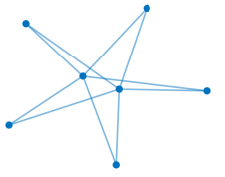

(a)

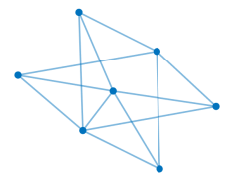

(b)

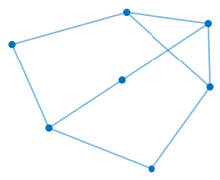

(c)

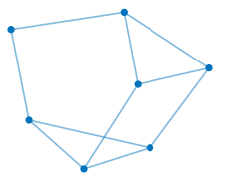

(d)

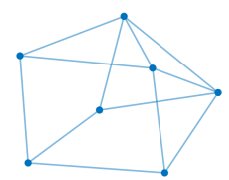

(e)

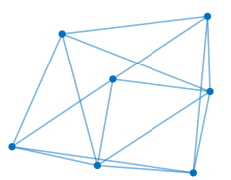

(f)

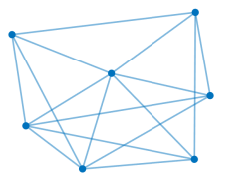

(g)

Figure 4. (a)-(g) Connected graphs with $N=7$ showing an "odd" behavior, i.e., having $Q_{A}<Q$. The graphs in (a)-(e) also have $\mu_{A, 2}<\mu_{2}$.

nodes. ER graphs were built by connecting each possible pair of nodes with a link probability $p$ [17]. In our simulations, we considered $p$ varying in the interval $p \in[0.1,0.9]$, eventually discarding unconnected graphs, thus generating graphs with different density and number of links. BA networks were constructed using the generative model described in [17], starting from $n_{0}=3$ fully connected nodes and adding at each growth step $m=2$ new links, connected by preferential attachment to the other already existing nodes of the network.

For each of these graphs, we have calculated the synchronizability measures, $\mu_{2}$ and $Q$ for connectivity based either on the classical, the hubs-attracting and the hubs-repelling Laplacian. The results are shown in Figure 5, confirming in both cases the theoretical expectations that coupling through the hubs-attracting Laplacian improves synchronizability with respect to the classical Laplacian (in particular, we may note that there are no cases where $\mu_{A, 2}<\mu_{2}$ or $\left.Q_{A}<Q\right)$. The theoretical bounds, provided by (20) for $\mu_{A, 2}$ and by (22) for $Q_{A}$, are also illustrated. The comparison with the corresponding synchronizability measures in the hubs-repelling Laplacian, namely $\mu_{R, 2}$ and $Q_{R}$, also reported in Figure 5, shows that, on the contrary, adopting a Laplacian implementing a hubs-repelling diffusion mechanism hinders synchronization; this is particularly critical in BA networks where both $\mu_{R, 2}$ and $Q_{R}$ are very small compared to $\mu_{A, 2}$ and $Q_{A}$.

A comparison of the values of $Q_{A}$ in ER and BA networks shows another manifestation of the heterogeneity paradox in synchronization: While heterogeneity in the degree distribution reduces the average distance between nodes, it also reduces the synchronizability of the network [28]. However, the hubs-attracting Laplacian mitigates the effect of heterogeneity, leading to an eigenratio $Q_{A}$ higher than $Q$, obtained with the classical Laplacian, in line with other results showing how weighting the links may result in improving the synchronizability of the original unweigthed topology [24].

Next, we investigated the question of whether the improvement in synchronizability obtained 
using the hubs-attracting Laplacian in place of the classical one is convenient in terms of the energy required to induce synchronization in the network. To compare the hubs-attracting Laplacian case with the classical Laplacian one, we selected for each of these two cases the minimum value of the coupling strength required to obtain synchronization, i.e., $\sigma^{A}=\frac{\alpha_{1}}{\mu_{A, 2}}$ and $\sigma=\frac{\alpha_{1}}{\mu_{2}}$, where $\alpha_{1}$ is defined as in (13). Then, as a proxy of energy associated to the network, we considered the Frobenius norm of the coupling matrix, which is given by the Laplacian times the coupling coefficient, i.e., $\|\sigma L\|_{F}=\sigma\|L\|_{F}=\sigma\left(\sum_{i, j} L_{i j}^{2}\right)^{\frac{1}{2}}$ and $\left\|\sigma^{A} L_{A}\right\|_{F}=\sigma^{A}\left(\sum_{i, j}\left(L_{A, i j}\right)^{2}\right)^{\frac{1}{2}}$. Their ratio is given by

$$
R=\frac{\sigma\|L\|_{F}}{\sigma^{A}\left\|L_{A}\right\|_{F}}=\frac{\mu_{A, 2}\|L\|_{F}}{\mu_{2}\left\|L_{A}\right\|_{F}}
$$

Figure 5(e)-(f) illustrates $R$ for the ER and BA networks considered in Figure 5 and compares it with $\frac{\|L\|_{F}}{\left\|L_{A}\right\|_{F}}$, representing the ratio between the energy associated to the two cases at $\sigma=1$. In most of the cases ( $100 \%$ for ER networks, $58 \%$ for BA networks), although the energy associated to the hubs-attracting Laplacian at $\sigma=1$ is higher than that of the classical Laplacian, the oppositive holds when the coupling coefficient is selected to achieve synchronization. This allows us to conclude that the improvement in the synchronizability does not occur at the expenses of a greater energy required to synchronize the network, but on the contrary is, in most cases, convenient in terms of energy.

5.2.3. Real-world networks. Finally, we have studied synchronizability of some real-world networks. In particular, we have examined a dataset of 54 real-world networks from different domains: ecological (includes food webs and ecosystems), social (networks of friendships, communication networks, corporate relationships), technological (internet, transport, software development networks), informational (vocabulary networks, citations), and biological (proteinprotein interaction networks, transcriptional regulation networks). The examples include networks with different numbers of nodes, ranging from $N=29$ to $N=4941$ nodes (for a more detailed description of the dataset and the characteristics of each network, please refer to [8]). For each of these networks, we have calculated the synchronizability parameters, $\mu_{2}$ and $Q$, for the classical, the hubs-attracting, and the hubs-repelling Laplacian connectivity cases.

The results of this analysis are shown in Figure 6 where we have now reported the relative change of the synchronizability parameters in $L_{A}$ with respect to their value in $L$, i.e., $\frac{\mu_{A, 2}-\mu_{2}}{\mu_{2}}$ (Figure 6(a)) and $\frac{Q_{A}-Q}{Q}$ (Figure 6(b)), and, similarly, for the hubs-repelling Laplacian $\frac{\mu_{R, 2}-\mu_{2}}{\mu_{2}}$ (Figure $6(\mathrm{c})$ ) and $\frac{Q_{R}-Q}{Q}$ (Figure $6(\mathrm{~d})$ ). We note that, generally, the connectivity based on the hubs-attracting Laplacian leads to a significant improvement in the synchronizability parameters, with four networks in particular displaying values of this improvement close or higher than 10. These are as follows: Canton $(N=108)$, Internet-1997 $(N=3015)$, Internet$1998(N=3522)$, and Stony $(N=112)$. For three networks we observe negative relative changes. These graphs are drugsA $(N=616)$; geom $(N=3621)$; softwaremysql $(N=1480)$. These three networks thus belong to the cases similar to those reported in Table 2, where the generic conditions $\mu_{A, 2} \geq \mu_{2}$ and $Q_{A} \geq Q$ are violated. We note, however, that the relative 
changes are in absolute value among the smallest observed in our dataset. On the contrary, for the hubs-repelling Laplacian we always observe negative relative changes, thus demonstrating that the adoption of this Laplacian deteriorates network synchronizability.

\section{Applications.}

6.1. Synchronization in phase oscillators. As an example of systems of coupled oscillators, here we consider the Kuramoto model on networks, where the units are purely phase oscillators. Each node is then associated to a scalar variable, $\theta_{i}(i=1, \ldots, N)$, parameterizing the motion along the limit cycle of the oscillator. In the Kuramoto model on networks, the dynamics of each unit is given by

$$
\dot{\theta}_{i}=\omega_{i}+\sigma \sum_{j=1}^{N} a_{i j} \sin \left(\theta_{j}-\theta_{i}\right),
$$

where $\omega_{i}$ is the natural oscillation frequency of unit $i$ and $\sigma$ the coupling coefficient. The model is here generalized to account for coupling regulated by hubs-attracting Laplacian:

$$
\dot{\theta}_{i}=\omega_{i}+\sigma \sum_{j=1}^{N} \frac{k_{j}}{k_{i}} a_{i j} \sin \left(\theta_{j}-\theta_{i}\right)
$$

In addition, in this section and in the following, for the sake of comparison we consider also coupling through the hubs-repelling Laplacian, which has been introduced in [8] and briefly recalled in section 3. The hubs-repelling Laplacian considers different weights for the links, such that high-degree nodes receive stronger influence from their neighbors, compared to low-degree ones. Furthermore, this influence is stronger if it comes from a lower degree node rather than a node with higher degree. Similarly to (25), the Kuramoto model on networks with hubs-repelling Laplacian reads

$$
\dot{\theta}_{i}=\omega_{i}+\sigma \sum_{j=1}^{N} \frac{k_{i}}{k_{j}} a_{i j} \sin \left(\theta_{j}-\theta_{i}\right) .
$$

Synchronization in networks (24), (25), and (26) is measured by the Kuramoto order parameter

$$
r=\left\langle\left|\frac{1}{N} \sum_{i=1}^{N} e^{\iota \theta_{i}}\right|\right\rangle_{T}
$$

where $\iota=\sqrt{-1}$ and \langle\rangle$_{T}$ denotes averaging over a window of time $T$ after discarding transient dynamics. The parameter $r$ takes values in $[0,1]$, with values close to one indicating synchronization, i.e., $\theta_{1}=\cdots=\theta_{N}$, while values close to zero are absent of coherence among the oscillators.

In the Kuramoto model, the higher algebraic connectivity the smaller the synchronization time and the critical value of the coupling coefficient over which synchronization is reached. 
Therefore, based on the properties of the hubs-attracting Laplacian discussed in section 5 and those of the hubs-repelling Laplacian illustrated in [8], it is expected that the use of the hubs-attracting Laplacian increases the synchronizability of a network with respect to the classical Laplacian case, whereas the hubs-repelling Laplacian decreases it. Our numerical results confirm these expectations. We illustrate them by considering two random networks with $N=100$ nodes and different topologies. The first is an ER graph generated with $p=0.06$ and having $M=286$ links; the second is a BA graph with $m=2$ links added per growth step, $n_{0}=3$ starting nodes, and $M=197$ links. We begin by illustrating the behavior of the order parameter $r$ with the coupling coefficient $\sigma$ (Figure $7(\mathrm{a})$ and (e)). To reach full synchronization, i.e., $r \simeq 1$, the network with the hubs-attracting Laplacian requires lower values of $\sigma$ than the classical Laplacian and the hubs-repelling one, a result reflecting that of $\mu_{A, 2}>\mu_{2}>\mu_{R, 2}$. We also note that, for the same value of $\sigma$, the higher value of $r$ occurs for the hubs-attracting Laplacian, followed then by the classical Laplacian and, finally, by the hubs-repelling Laplacian that has the lower value of $r$.

Next, we study the synchronized clusters that form for increasing values of $\sigma$. To this aim, we follow the approach described in [10], by defining a new matrix $\mathcal{D}$ with elements given by $\mathcal{D}_{i j}=a_{i j}\left|\left\langle e^{\iota\left(\theta_{i}(t)-\theta_{j}(t)\right)}\right\rangle_{T}\right|$, applying a threshold $\delta=0.8$, such that those pairs of nodes for which $\mathcal{D}_{i j}>\delta$ are considered to be synchronized, and finally calculating the synchronous clusters that form at different values of $\sigma$. We have considered a series of quantities of interest such as the size of the giant component, $G C$, of these synchronous clusters, the average degree of the nodes in the connected components, $\left\langle k_{i}\right\rangle_{C C}$, and the number of hubs in the connected components, $h_{C C}$. Each of these parameters is a function of the coupling coefficient $\sigma$ as well as $r$ is, so that we have plotted each of them as a function of the corresponding value of $r$ obtained for the given $\sigma$.

The result of this analysis is shown in Figure 7(b)-(d) for the ER graph and in Figure $7(\mathrm{f})$-(h) for the BA graph. The different values of each of these parameters in correspondence of the same value of $r$ indicate that the diverse coupling mechanism in the three cases leads to synchronous clusters with different characteristics. Equivalently, one can observe that the same average level of synchronization, measured by $r$, is obtained in the three cases with clusters with different characteristics. For low $r$ the giant component is larger in the hubsattracting Laplacian coupling scheme than in the classical and in the hubs-repelling ones, while for higher $r$ the opposite occurs in the BA graph, while giant components of similar sizes are obtained in the ER graph. The synchronous clusters that form at low $r$ involve more hubs in the case of the classical and hubs-attracting Laplacian rather than for the hubsrepelling Laplacian, thus also resulting in a higher $\left\langle k_{i}\right\rangle_{C C}$. All in all, these results point in the direction of hallmarking a different role of the hubs in the diverse coupling schemes; they likely act as collectors of information in the case of hubs-repelling Laplacian and as spreaders of information in the hubs-attracting Laplacian scheme, thus promoting in the latter case the birth at low $r$ of synchronous clusters around the hubs. An exemplificative illustration of the different synchronous clusters obtained in the BA graph at $r \simeq 0.2$ for the three diverse coupling schemes is shown in Figure 8, which highlights the presence of structures centered around the hubs in the hubs-attracting Laplacian case and clustering several nodes, whereas in comparison smaller clusters appear for the two other coupling schemes. 
6.2. Formation control in unicycles. The second application we consider is formation control, where among the different models used for the dynamics of the agents, the simplest one, able to account for the kinetic of a mobile robot, is given by the unicycles model [21]. The coordinates of the unicycles in the complex plane are represented by $r_{i}(t)=y_{1, i}(t)+\iota y_{2, i}(t)$, for $t \geq 0$.

The dynamics of the position of the unicycle in Cartesian coordinates is given by $\dot{y}_{1, i}(t)=$ $v_{i} \cos \theta_{i}(t)$ and $\dot{y}_{2, i}(t)=v_{i} \sin \theta_{i}(t)$, and $\dot{\theta}_{i}(t)=\omega_{i}(t)$, where $v_{i}$ is the speed of the unicycle, and $\omega_{i}(t)$ is the angular velocity used as the control input for the system. A schematic representation of a unicycle is reported in Figure 9.

Let us consider a group of $N$ unicycles interacting through the different coupling schemes based on the classical, hubs-attracting, and hubs-repelling Laplacian. Formation control is achieved by considering as control law $\omega_{i}(t)=\sum_{j=1}^{N} a_{i j} \sin \left(\theta_{j}(t)-\theta_{i}(t)\right)$ (classical Laplacian), $\omega_{i}(t)=\sum_{j=1}^{N} \frac{k_{j}}{k_{i}} a_{i j} \sin \left(\theta_{j}(t)-\theta_{i}(t)\right)$ (hubs-attracting Laplacian), or $\omega_{i}(t)=\sum_{j=1}^{N} \frac{k_{i}}{k_{j}} a_{i j} \sin \left(\theta_{j}(t)\right.$ $-\theta_{i}(t)$ ) (hubs-repelling Laplacian), so that the dynamics of the unicycles are governed by

$$
\dot{\theta}_{i}(t)=\sum_{j=1}^{N} a_{i j} \sin \left(\theta_{j}(t)-\theta_{i}(t)\right)
$$

for the classical Laplacian case, or

$$
\dot{\theta}_{i}(t)=\sum_{j=1}^{N} \frac{k_{j}}{k_{i}} a_{i j} \sin \left(\theta_{j}(t)-\theta_{i}(t)\right)
$$

for the hubs-attracting Laplacian case, or

$$
\dot{\theta}_{i}(t)=\sum_{j=1}^{N} \frac{k_{i}}{k_{j}} a_{i j} \sin \left(\theta_{j}(t)-\theta_{i}(t)\right)
$$

for the hubs-repelling Laplacian case. For the purpose of illustration and without lack of generality, we fix an arbitrary network of $N=10$ agents (similar results are obtained for larger networks and different topologies). The network is shown in the panel of Figure 10(a), its classical, hubs-attracting, and hubs-repelling Laplacians are given by

$$
L=\left(\begin{array}{cccccccccc}
3 & 0 & 0 & 0 & 0 & -1 & -1 & 0 & 0 & -1 \\
0 & 3 & 0 & 0 & -1 & -1 & -1 & 0 & 0 & 0 \\
0 & 0 & 5 & 0 & -1 & -1 & -1 & -1 & 0 & -1 \\
0 & 0 & 0 & 2 & 0 & 0 & 0 & 0 & -1 & -1 \\
0 & -1 & -1 & 0 & 3 & 0 & 0 & 0 & 0 & -1 \\
-1 & -1 & -1 & 0 & 0 & 5 & 0 & -1 & -1 & 0 \\
-1 & -1 & -1 & 0 & 0 & 0 & 3 & 0 & 0 & 0 \\
0 & 0 & -1 & 0 & 0 & -1 & 0 & 2 & 0 & 0 \\
0 & 0 & 0 & -1 & 0 & -1 & 0 & 0 & 2 & 0 \\
-1 & 0 & -1 & -1 & -1 & 0 & 0 & 0 & 0 & 4
\end{array}\right)
$$




$$
\begin{aligned}
& L_{A}=\left(\begin{array}{cccccccccc}
4 & 0 & 0 & 0 & 0 & -1.67 & -1 & 0 & 0 & -1.33 \\
0 & 3.67 & 0 & 0 & -1 & -1.67 & -1 & 0 & 0 & 0 \\
0 & 0 & 3.40 & 0 & -0.60 & -1 & -0.60 & -0.40 & 0 & -0.80 \\
0 & 0 & 0 & 3 & 0 & 0 & 0 & 0 & -1 & -2 \\
0 & -1 & -1.67 & 0 & 4 & 0 & 0 & 0 & 0 & -1.33 \\
-0.60 & -0.60 & -1 & 0 & 0 & 3 & 0 & -0.40 & -0.40 & 0 \\
-1 & -1 & -1.67 & 0 & 0 & 0 & 3.67 & 0 & 0 & 0 \\
0 & 0 & -2.50 & 0 & 0 & -2.50 & 0 & 5 & 0 & 0 \\
0 & 0 & 0 & -1 & 0 & -2.50 & 0 & 0 & 3.50 & 0 \\
-0.75 & 0 & -1.25 & -0.50 & -0.75 & 0 & 0 & 0 & 0 & 3.25
\end{array}\right) ; \\
& L_{R}=\left(\begin{array}{cccccccccc}
2.35 & 0 & 0 & 0 & 0 & -0.60 & -1 & 0 & 0 & -0.75 \\
0 & 2.60 & 0 & 0 & -1 & -0.60 & -1 & 0 & 0 & 0 \\
0 & 0 & 8.09 & 0 & -1.67 & -1 & -1.67 & -2.50 & 0 & -1.25 \\
0 & 0 & 0 & 1.50 & 0 & 0 & 0 & 0 & -1 & -0.50 \\
0 & -1 & -0.60 & 0 & 2.35 & 0 & 0 & 0 & 0 & -0.75 \\
-1.67 & -1.67 & -1 & 0 & 0 & 9.34 & 0 & -2.50 & -2.50 & 0 \\
-1 & -1 & -0.60 & 0 & 0 & 0 & 2.60 & 0 & 0 & 0 \\
0 & 0 & -0.40 & 0 & 0 & -0.40 & 0 & 0.80 & 0 & 0 \\
0 & 0 & 0 & -1 & 0 & -0.40 & 0 & 0 & 1.40 & 0 \\
-1.33 & 0 & -0.80 & -2 & -1.33 & 0 & 0 & 0 & 0 & 5.46
\end{array}\right) ;
\end{aligned}
$$

and we have $\mu_{2}=0.9962, \mu_{A, 2}=1.9560, \mu_{R, 2}=0.4757$. We analyze the behavior of the multiagent system in the three coupling cases by monitoring the Kuramoto order parameter (27) which represents the level of coherence in the heading coordination. Formation control corresponds to $r \simeq 1$.

In Figure 10(a), $r$ is reported as a function of time $t$; full coordination is reached in the three cases but with a different dynamics. In particular, if interactions are ruled by the hubs-attracting Laplacian, the system is faster than in the other two cases, with the classical Laplacian case representing the intermediate behavior between the two extremes. In Figure 10 (b)-(d) the trajectories of the unicycles on the plane $y_{1}-y_{2}$ are shown, from $t=0$ (red circles) to $t=8$ (blue arrows); interactions are ruled by the classical Laplacian in Figure 10(b), the hubs-attracting Laplacian in Figure 10(c), and the hubs-repelling one in Figure 10(d). When the interactions are through the hubs-repelling Laplacian, the agents slowly converge to a common heading, while in the other two cases the units reach synchronization in a shorter time, with the hubs-attracting Laplacian coupling scheme representing the most efficient case. 

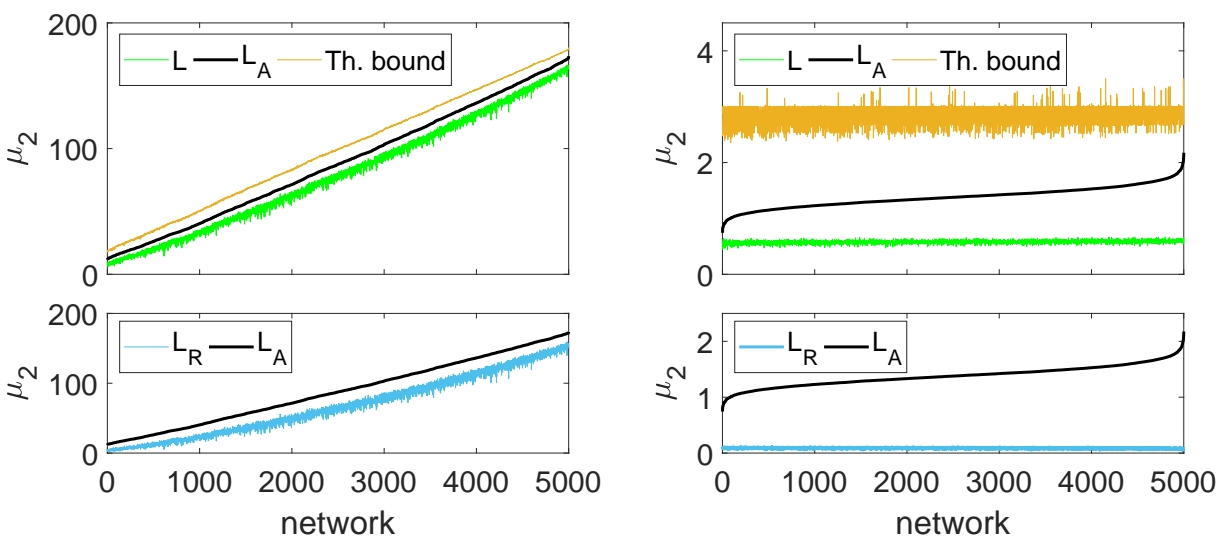

(a)

(b)
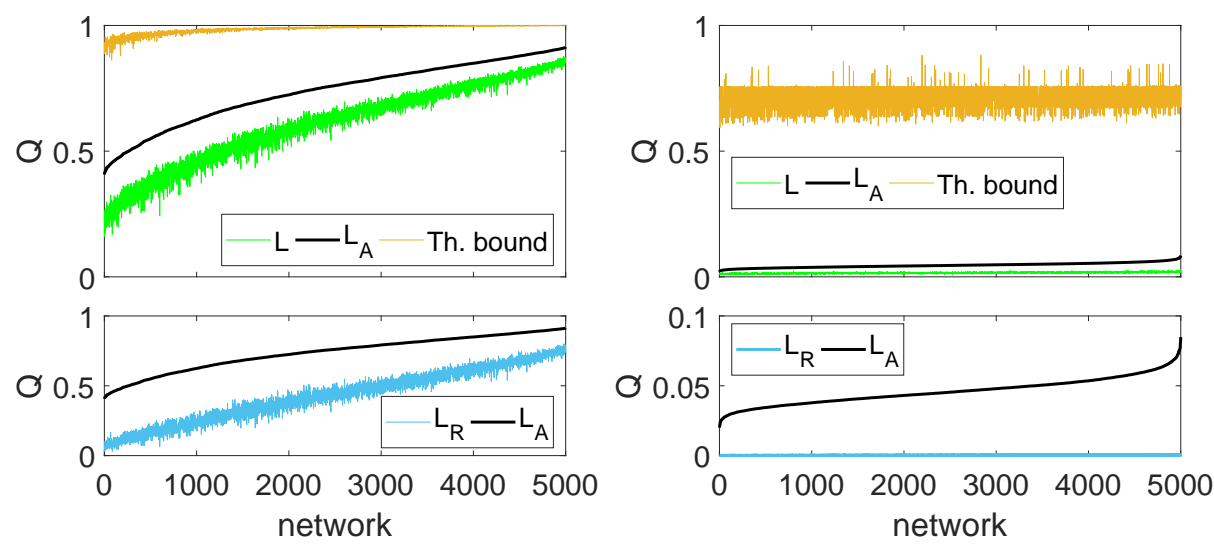

(c)

(d)

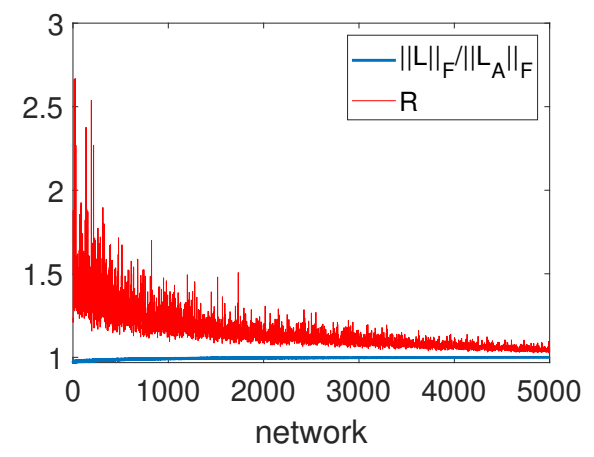

(e)

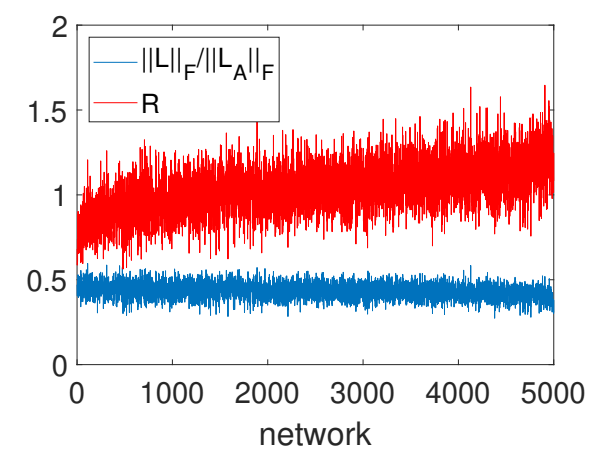

(f)

Figure 5. Synchronizability of 5,000 networks with $N=200$ nodes based on classical or hubs-attracting Laplacians (a), (c), (e) ER model; (b), (d), (f) BA model. (a)-(b) Algebraic connectivity $\mu_{2}$. (c)-(d) Eigenratio $Q$. (e)-(f) Ratio between the energy associated to classical Laplacian and hubs-attracting Laplacian at $\sigma=1$ and parameter $R$. The theoretical bounds are provided by (20) for $\mu_{A, 2}$ and by (22) for $Q_{A}$. For the latter, when this bound is trivial, i.e., $Q_{A}>1$, we simply give the nontrivial upper bound of $Q_{A}=1$ in the plot. For better visualization networks are reordered according to increasing values of $\mu_{A, 2}$ in (a), (b), (e), (f) or $Q_{A}$ in (c) and $(\mathrm{d})$.

Copyright (C) by SIAM. Unauthorized reproduction of this article is prohibited. 


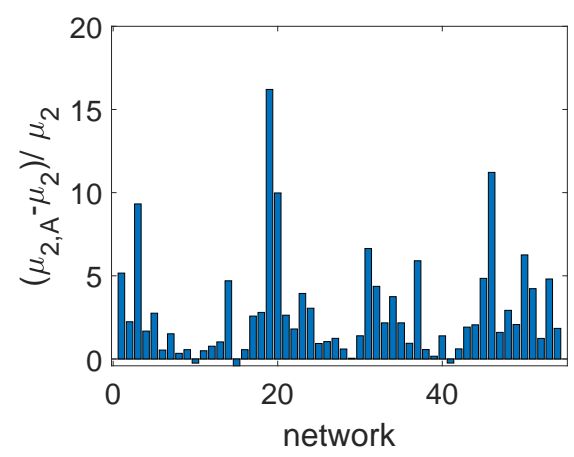

(a)

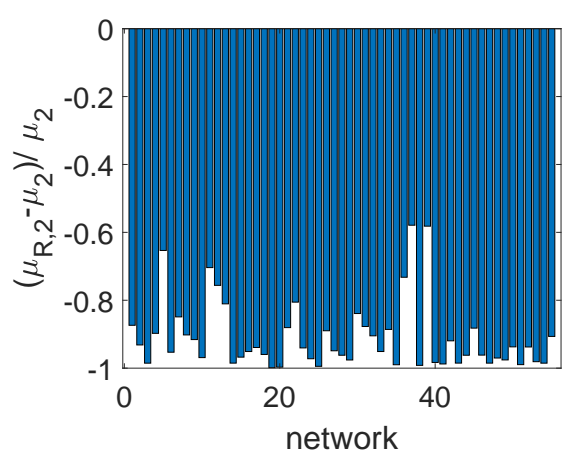

(c)

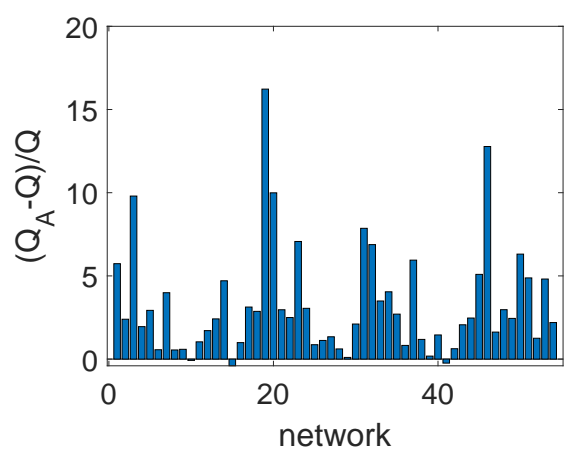

(b)

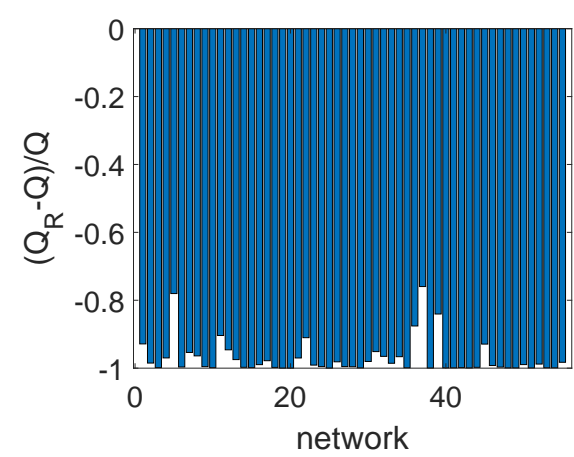

(d)

Figure 6. Relative change of the synchronizability parameters in $L_{A}$ with respect to their value in $L$ : (a) $\frac{\mu_{A, 2}-\mu_{2}}{\mu_{2}}$; (b) $\frac{Q_{A}-Q}{Q}$; and in $L_{R}$ with respect to their value in $L$ : (c) $\frac{\mu_{R, 2}-\mu_{2}}{\mu_{2}}$; (b) $\frac{Q_{R}-Q}{Q}$. 


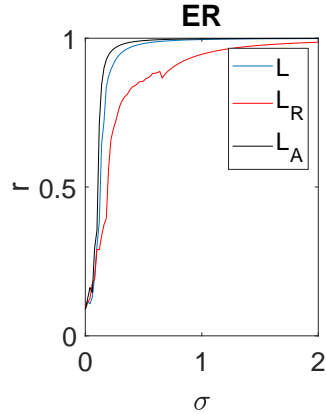

(a)

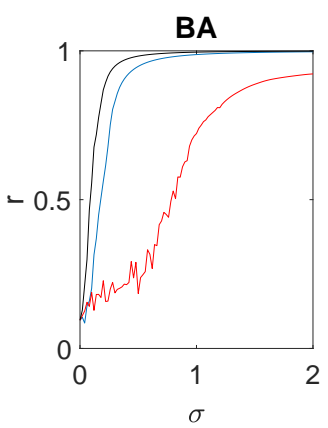

(e)

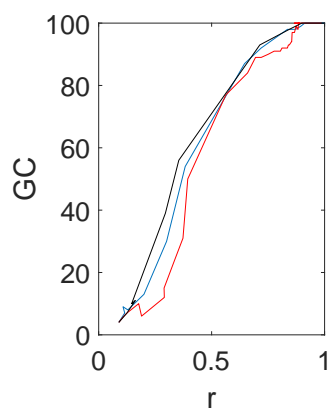

(b)

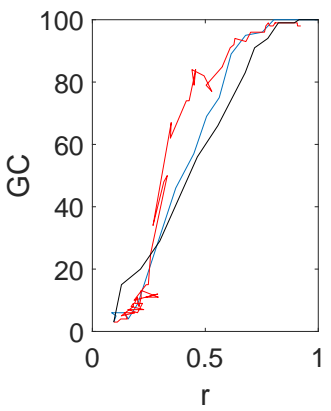

(f)

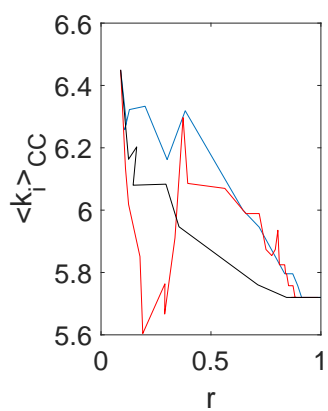

(c)

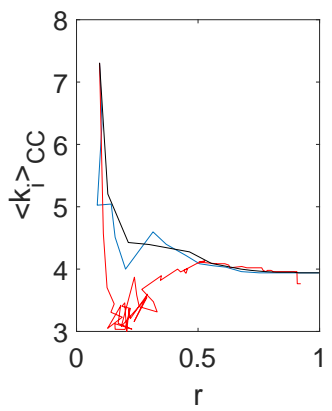

(g)

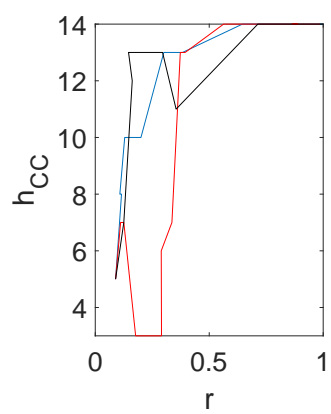

(d)

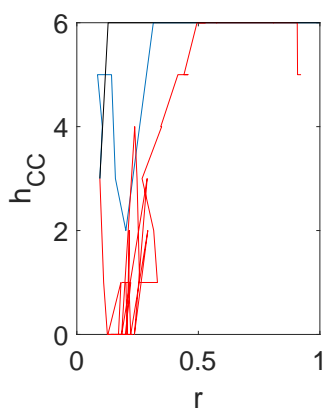

(h)

Figure 7. Synchronization in networks of phase oscillators coupled through classical, hubs-attracting, or hubs-repelling Laplacian. (a), (e) Order parameter $r$. (b), (f) Size of the giant component with respect to $r$. (c), (g) Average degree for the nodes in the connected components with respect to $r$. (d), (h) Number of hubs in the connected components with respect to $r$. The results refer to an ER network, (a)-(d), or to a BA network (e)-(h).

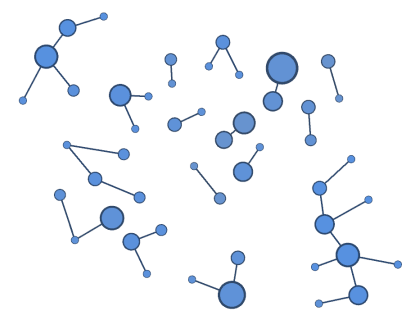

(a)

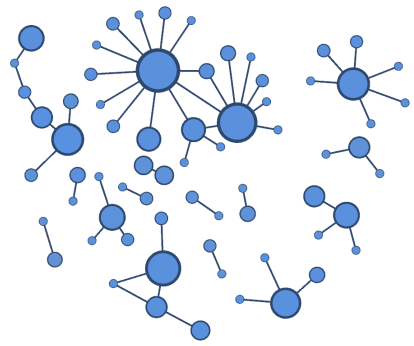

(b)

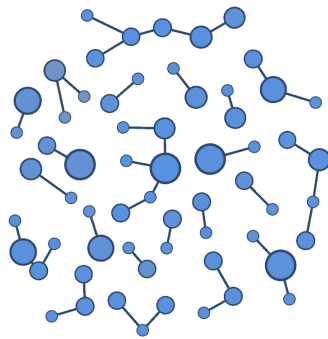

(c)

Figure 8. Synchronous clusters obtained in the BA graph at $r \simeq 0.2$ for coupling schemes based on (a) classical Laplacian; (b) hubs-attracting Laplacian; (c) hubs-repelling Laplacian. The size of the nodes is proportional to their degree in the original network.

Copyright ( $\odot$ by SIAM. Unauthorized reproduction of this article is prohibited. 


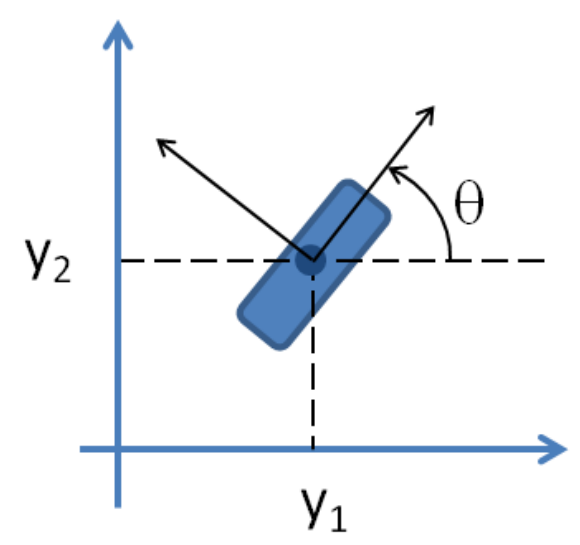

Figure 9. Scheme of the unicycle.

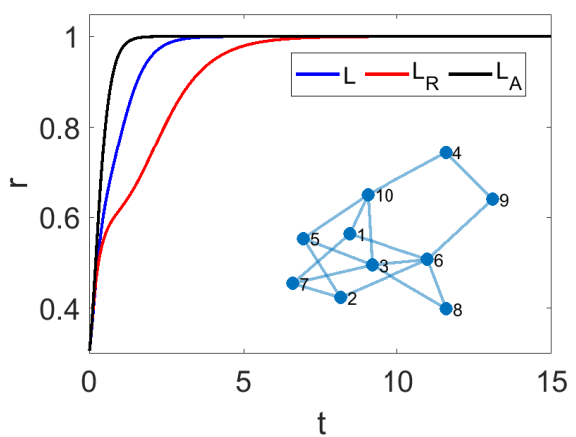

(a)

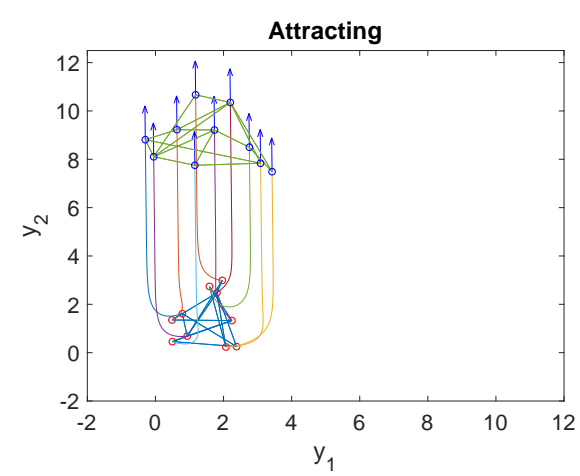

(c)

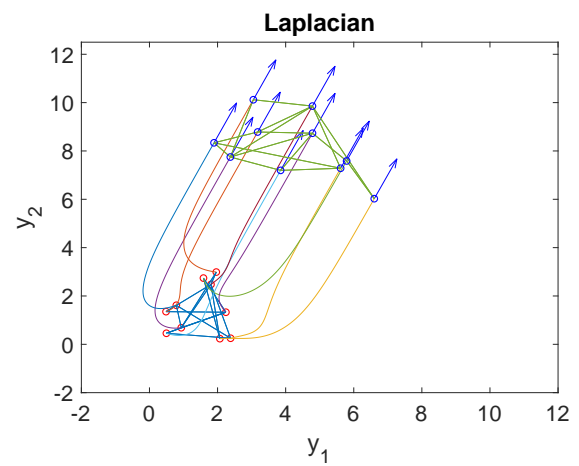

(b)

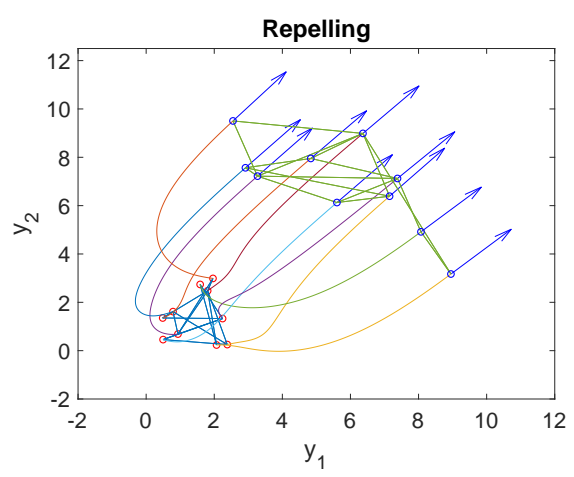

(d)

Figure 10. Behavior of a group of $N=10$ unicycles: (a) Kuramoto order parameter $r$ versus time $t$ for the three Laplacians considered; trajectory of the group in the plane $y_{1}-y_{2}$ in the case of (b) classical Laplacian, (c) hubs-attracting Laplacian, and (d) hubs-repelling Laplacian. The network of interaction is shown at the start and end point of the trajectory.

Copyright $\odot$ by SIAM. Unauthorized reproduction of this article is prohibited. 
7. Conclusions. In this work, we have defined the hubs-attracting Laplacian, studied the properties of its spectrum through analytical and computational results, and related them to synchronization processes occurring in the network. The hubs-attracting Laplacian is nonsymmetric, but we proved that its eigenvalues are real. This property is relevant as it allows one to straightforwardly generalize the synchronizability measures used for coupling based on the classical Laplacian. The study of these measures shows that coupling through the hubs-attracting Laplacian yields better synchronization properties for most of the cases, where the graphs for which an improvement is not observed are found to occur with low probability. The characterization of these graphs is a possible future direction of this study. Our analytical results also provide bounds for the synchronizability measures allowing one to estimate the maximum theoretical enhancement that can be derived from the use of the hubs-attracting Laplacian. Finally, we have studied two specific models of units coupled through the hubs-attracting Laplacian. The first one is based on phase oscillators and, quite interestingly, shows how the same level of synchronization is reached through different synchronous clusters when the coupling mechanism is varied from classical to hubs-repelling and hubs-attracting Laplacian. A further deeper characterization of these structures and the microscopic mechanisms leading to them is another possible direction for future work. The second example refers to coordination of unicycles, providing an idealized case study where the use of the hubs-attracting Laplacian is beneficial and paving the way to other applications that may benefit from communication protocols based on this Laplacian.

\section{REFERENCES}

[1] A. Arenas, A. Díaz-Guilera, J. Kurths, Y. Moreno, and C. Zhou, Synchronization in complex networks, Phys. Rep., 469 (2008), pp. 93-153.

[2] S. Boccaletti, V. Latora, Y. Moreno, M. Chavez, and D.-U. Hwang, Complex networks: Structure and dynamics, Phys. Rep., 424 (2006), pp. 175-308.

[3] M. Bonaventura, V. Nicosia, And V. Latora, Characteristic times of biased random walks on complex networks, Phys. Rev. E, 89 (2014), 012803.

[4] Z. Burda, J. Duda, J.-M. LuCK, AND B. WAClaw, Localization of the maximal entropy random walk, Phys. Rev. Lett., 102 (2009), 160602.

[5] G. Chen And Z. DuAn, Network synchronizability analysis: A graph-theoretic approach, Chaos: An Interdisciplinary Journal of Nonlinear Science, 18 (2008), 037102.

[6] Z. Duan, G. Chen, And L. Huang, Complex network synchronizability: Analysis and control, Phys. Rev. E, 76 (2007), 056103.

[7] Z. Duan, C. LiU, And G. Chen, Network synchronizability analysis: The theory of subgraphs and complementary graphs, Phys. D, 237 (2008), pp. 1006-1012.

[8] E. EstradA, Hubs-repelling' Laplacian and related diffusion on graphs/networks, Linear Algebra Appl., 596 (2020), pp. 256-280.

[9] A. Gerbaud, K. Altisen, S. Devismes, and P. Lafourcade, Comparison of mean hitting times for a degree-biased random walk, Discrete Appl. Math., 170 (2014), pp. 104-109.

[10] J. Gómez-Gardenes, Y. Moreno, And A. Arenas, Paths to synchronization on complex networks, Phys. Rev. Lett., 98 (2007), 034101.

[11] R. Grone And R. MerRis, The Laplacian spectrum of a graph II, SIAM J. Discrete Math., 7 (1994), pp. $221-229$.

[12] R. Grone, R. Merris, And V. S. Sunder, The Laplacian spectrum of a graph, SiAM J. Matrix Anal. Appl., 11 (1990), pp. 218-238, https://doi.org/10.1137/0611016.

[13] A. Hagberg And D. A. Schult, Rewiring networks for synchronization, Chaos: An Interdisciplinary 
Journal of Nonlinear Science, 18 (2008), 037105.

[14] H. Hong, B. J. Kim, M. ChoI, AND H. PARK, Factors that predict better synchronizability on complex networks, Phys. Rev. E, 69 (2004), 067105.

[15] L. Huang, Q. Chen, Y.-C. LaI, And L. M. Pecora, Generic behavior of master-stability functions in coupled nonlinear dynamical systems, Phys. Rev. E, 80 (2009), 036204.

[16] L. Kempton, G. Herrmann, and M. Di Bernardo, Self-organization of weighted networks for optimal synchronizability, IEEE Trans. Control Netw. Syst., 5 (2017), pp. 1541-1550.

[17] V. Latora, V. Nicosia, And G. Russo, Complex Networks: Principles, Methods, and Applications, Cambridge University Press, Cambridge, 2017.

[18] J. Lu, X. Yu, G. Chen, And D. Cheng, Characterizing the synchronizability of small-world dynamical networks, IEEE Trans. Circuits Syst. I. Regul. Pap., 51 (2004), pp. 787-796.

[19] N. Masuda, M. A. Porter, And R. Lambiotte, Random walks and diffusion on networks, Phys. Rep., 716 (2017), pp. 1-58.

[20] R. MerRis, Laplacian matrices of graphs: A survey, Linear Algebra Appl., 197 (1994), pp. 143-176.

[21] M. Mesbahi And M. Egerstedt, Graph Theoretic Methods in Multiagent Networks, Vol. 33, Princeton University Press, Princeton, NJ, 2010.

[22] B. Mohar, The Laplacian Spectrum of Graphs, in Graph Theory, Combinatorics, and Applications, Vol. 2, Y. Alavi, G. Chartrand, and O. Oellermann, eds., Wiley, New York, 1991.

[23] R. J. Mondragón, Core-biased random walks in networks, J. Complex Netw., 6 (2018), pp. $877-886$.

[24] A. E. Motter, C. Zhou, AND J. Kurths, Network synchronization, diffusion, and the paradox of heterogeneity, Phys. Rev. E, 71 (2005), 016116.

[25] D. Mugnolo, Semigroup Methods for Evolution Equations on Networks, Springer, Cham, 2014.

[26] T. Nishikawa AND A. E. Motter, Synchronization is optimal in nondiagonalizable networks, Phys. Rev. E, 73 (2006), 065106.

[27] T. Nishikawa AND A. E. MotTer, Network synchronization landscape reveals compensatory structures, quantization, and the positive effect of negative interactions, Proc. Natl. Acad. Sci. USA, 107 (2010), pp. 10342-10347.

[28] T. Nishikawa, A. E. Motter, Y.-C. Lai, and F. C. Hoppensteadt, Heterogeneity in oscillator networks: Are smaller worlds easier to synchronize?, Phys. Rev. Lett., 91 (2003), 014101.

[29] R. Pastor-Satorras, C. Castellano, P. Van Mieghem, and A. Vespignani, Epidemic processes in complex networks, Rev. Modern Phys., 87 (2015), pp. 925-979.

[30] L. M. Pecora And T. L. Carroll, Master stability functions for synchronized coupled systems, Phys. Rev. Lett., 80 (1998), .2109.

[31] C. Poignard, T. Pereira, And J. P. Pade, Spectra of Laplacian matrices of weighted graphs: Structural genericity properties, SIAM J. Appl. Math., 78 (2018), pp. 372-394, https://doi.org/10. $1137 / 17 \mathrm{M} 1124474$.

[32] A. A. RAD, M. JALILI, AND M. HASLER, Efficient rewirings for enhancing synchronizability of dynamical networks, Chaos: An Interdisciplinary Journal of Nonlinear Science, 18 (2008), 037104.

Copyright ( $\odot$ by SIAM. Unauthorized reproduction of this article is prohibited. 